\title{
Anthocyanin-loaded polymeric dextran nanoparticle as an anti- proliferative agent on human liver carcinoma cells
}

\author{
Siti Zubaidah Abdullah ${ }^{\mathrm{a}}$, Chean Ring Leong ${ }^{\mathrm{a}}$, Woei Yenn Tong ${ }^{\mathrm{a}^{*}}$, Jyh Chyang Pang ${ }^{\mathrm{b}}$, Wen-Nee Tan ${ }^{\mathrm{c}}$, \\ Syarifah Ab Rashid ${ }^{a}$ \\ ${ }^{a}$ Universiti Kuala Lumpur, Branch Campus Malaysian Institute of Chemical and Bioengineering Technology, Lot 1988 Kawasan Perindustrian \\ Bandar Vendor, Taboh Naning, 78000 Alor Gajah, Melaka, Malaysia \\ ${ }^{b}$ Universiti Kuala Lumpur, Branch Campus Institute of Medical Science Technology, A1-1 Jalan TKS 1,Taman Kajang Sentral, 43000 Kajang, \\ Selangor, Malaysia \\ ${ }^{c}$ Chemistry Section, School of Distance Education, Universiti Sains Malaysia, Minden, Penang, Malaysia
}

Received 7th May 2020 / Accepted 28th July 2020

\begin{abstract}
In this communication, anthocyanin-loaded dextran nanoparticles from Clitoria ternatea was synthesized and characterized to test its anti-proliferative activity on the human HepG2 liver cancer cell lines. By using dextran as an encapsulant polymer, the nanoparticles appeared to be spherical, with an average size of $45.5 \pm 11 \mathrm{~nm}$. The surface charge of the anthocyanin-loaded dextran nanoparticle was $4.39 \mathrm{mV}$, which slightly relative to free anthocyanin $(-4.46 \mathrm{mV})$, which indicate good dispersion stabilities. The Fourier transform infrared analysis showed that the anthocyanins from C. ternatea was successfully encapsulated in dextran nanoparticles. Overall, the percentage of drug encapsulation efficiency was 3.03\%. Based on the stability test, the anthocyanin-loaded dextran nanoparticle showed significantly better color stability index compared to free anthocyanin, particularly at the presence of light and temperature of $37^{\circ} \mathrm{C}$ and $50^{\circ} \mathrm{C}$. In the anti-proliferation assay on HepG2 liver cancer cell lines, the viability of the cancer cells was significantly reduced after treatment with the anthocyanin-loaded dextran nanoparticle. The antiproliferation activities of the nanoparticles were significantly better than free anthocyanin. Our findings revealed the ability of the anthocyanin-loaded dextran nanoparticle, in particular from C. ternatea, as an effective anti-proliferative agent against cancer cells. Nanoencapsulation with dextran significantly improve the efficacy and stability of the anthocyanins. Further investigations should be done to evaluate the in vivo efficacy.
\end{abstract}

Keywords: Anthocyanin nanoparticle, anti-proliferative activity, Clitoria ternatea, nano-encapsulation

\section{INTRODUCTION}

Cancer is a disease characterized by unregulated proliferation of cells, which results in the abnormal cells formation and uncontrolled growth of tissue. Globally, cancer is recognized as the second foremost cause of death and is expected to root an additional 9.6 million deaths in 2018. To date, about one in six deaths are due to cancer (Bray et al., 2018). Surgical resection, radiation or chemotherapy are among the standard protocol of treatments for cancer patients (Windchester et al., 2010). However, the success rate for the cancer treatment is low as current treatments are often implicated with poor response, low survival rate and development of

*Author for correspondence: Tong Woei Yenn, Universiti Kuala Lumpur, Branch Campus Malaysian Institute of Chemical and Bioengineering Technology, Lot 1988 Kawasan Perindustrian Bandar Vendor, Taboh Naning, 78000 Alor Gajah, Melaka, Malaysia.Email - wytong@unikl.edu.my 
metastatic cells. Thus, there is a need to explore new approaches for the treatment of cancer (Zununi et al., 2019).

Clitoria ternatea or blue pea can be found in Asian countries. It is a perennial climber crop native (Bhatia et al., 2014) and rich with pigments, especially anthocyanins. The chemical exists in flavylium salts as their natural form (Kong et al., 2003) and gives a color variety, including bright red, purple and blue, to fruits and vegetables (Wang et al., 2008). Anthocyanins have been shown to give benefits in visual acuity, cancer, heart disease and age-related neurodegenerative disorders (Ray et al., 2009). Besides, the epidemiologic studies suggested the anti-oxidant, anti-microbial and anti-inflammatory activities of anthocyanins can lower the risk of cardiovascular disease, diabetes, arthritis and cancer (Prior and Wu, 2006; Thanh et al., 2020). Hwang et al. (2011) reported that anthocyanins can boost memory and the anti-carcinogen activities of the anthocyanins are competent to protect the liver.

The phenolic structure of anthocyanin has been associated with the antioxidant activity including the ability to scavenge superoxide, singlet oxygen, peroxide, hydrogen peroxide, and bydroxyl radical. These substances are categorized as reactive oxygen species (ROS) (Wang \& Jiao, 2000). The antioxidant efficiency of anthocyanins was previously verified in many cell culture systems i.e. colon, endothelial, liver, breast, leukemic cells, and keratinocytes (Renis et al., 2008; Parry et al., 2006; Shih et al., 2007; Yang et al., 2019). Anthocyanins have demonstrated several anti-toxic and anti-carcinogenic properties by scavenging reactive oxygen species in the cell culture system, thereby enhancing the oxygenradical absorbing ability of cells (Yang et al., 2019). Therefore, the protective effects of anthocyanins are rather sensitive towards high temperature and light condition with relatively shorter viable shelf life (Karaoglan et al., 2019; Gerald et al., 2019). Nano-encapsulation could be a new approach to prolong the viable shelf life of the protective effects of anthocyanins in the context of cancer treatment or even prevention.

Nanoparticles are solid particle with a size range of 10 to $200 \mathrm{~nm}$ (Khan et al., 2019). Nanoparticles can be obtained via various preparation processes. The drug is dissolved and encapsulated or bound to a nanoparticle matrix as a delivery medium. Drug-incorporated matrix nanoparticle is important to attain the site-specific action by controlling the particle size, surface properties and releases of the targeted drug (Mohanraj \& Chen, 2006). Nanoparticle is a key aspect of nanomedicines whereby anticancer therapeutic drugs are encapsulated inside a fatty matrix of a suitable size for delivery whilst avoiding systemic side-effects (Khan et al., 2019). Such a particle-matrix will allow sustained release of the chemotherapeutic over a long time scale and minimizing systemic side effects. Besides, the type of polymer used can be designed to deliver the therapeutic agent to the site specific targets. The polymers used including polyhydroxyalkanoate (PHA), poly-lactide-coglycolide (PLGA), cyclodextrins and dextrans. Dextran is widely used for anticancer drug delivery purpose due to its excellent biocompatibility, non-toxic and nonimmunogenic properties. The anticancer efficiency and selectivity of Goniothalamin can be enhaced by encapsulating the drug with $\mathrm{pH}$ sensitive acetalated dextran nanoparticles (Braga et al., 2020). Besides, dextran was also combined with polyacrylamide as matrices to create an anticancer nanocomposite (Telegeev et al., 2017). The anticancer properties of dextran-cathecin and dextran-quercetin conjugates were also reported (Vittorio et al., 2016; Oliver et al., 2018).

In this study, in order to improve the anticancer properties and stability of $C$. ternatea derived anthocyanins, the nanoparticles were synthesized using dextran as an encapsulant. Synthesis and characterization of anthocyaninloaded polymeric dextran nanoparticle were investigated. Moreover, its anti-proliferative activity was tested on human liver carcinoma cells, HepG2, in an attempt for the exploratory development of anthocyanin as a potential anticancer therapeutic approach.

\section{MATERIALS AND METHODS}

\section{Plant sources}

C. ternatea flowers, with no visible diseases, were collected at Jalan Tengkera (N 2० $12^{\prime} 3^{\prime \prime} \mathrm{E} 102^{\circ}$ 14' 21"), Bandaraya Melaka, Melaka, Malaysia. The samples were kept in ziplock bags, prior to 
the cleaning process under flowing tap water. This process was done within $24 \mathrm{~h}$ after collection. Then, the flowers were dried in an incubator at $60^{\circ} \mathrm{C}$ until stable weight achieved. The dried flowers were ground into fine powder form by using a food blender (Panasonic, Japan). This powder was kept in a desiccator before further use.

\section{Preparation of anthocyanin extract}

In order to prepare an acidified ethanol, $1 \mathrm{M}$ of acetic acid (Acros Organics, USA) was added to ethanol (Fisher Scientific, USA) until pH 4.5 was achieved. Next, $1 \mathrm{~g}$ plant powder was soaked in $20 \mathrm{~mL}$ acidified ethanol for 3 days. This mixture was stirred periodically. Then, the crude extract paste was obtained by drying the liquid extract under reduced pressure through a rotary evaporator. The temperature was set at $50^{\circ} \mathrm{C}$. The anthocyanin test was performed by applying the extract to $2 \mathrm{~N}$ hydrochloric acid (Acros Organics, USA) and ammonia solution (Acros Organics, USA) at equal volume $(\mathrm{v} / \mathrm{v})$. The presence of the blue-violet solution denotes a positive result for anthocyanin (Leong et al., 2017).

\section{Synthesis of anthocyanin-loaded dextran nanoparticles}

Hundred microliter of 2\% of pluronic F127 (Sigma-Aldrich, USA) solution was prepared in distilled water in an ice bath. After that, $200 \mathrm{~mL}$ of $1 \%$ anthocyanin extract was added into the aforementioned solution. Then, it was blended with a homogenizer (Heidolph, Germany) at $10,000 \mathrm{rpm}$ for $2 \mathrm{~min}$. Subsequently, $1 \mathrm{~g}$ of dextran (R\&M, India) was mixed into the homogenized solution and further blended for 1 $\min$ at $10,000 \mathrm{rpm}$ until it changed into transparent. This solution was subjected to a freeze dryer (Labconco, USA) and left to dry for 48 h. A blank control was integrated by substituting the anthocyanin extract with ethanol (Tan et al. 2019).

\section{Microscopic characterization}

The ultra-structures of the anthocyanin-loaded dextran nanoparticles were observed under transmission electron microscopy (TEM) (Philips CM12, Netherlands). By using a clean dropper, one droplet of anthocyanin-loaded dextran nanoparticle solution was put on a carbon grafted copper grid, prior to staining procedure by uranyl acetate. Then, the sample was dried at ambient temperature and viewed under TEM (Tong et al., 2017).

\section{Surface charge measurement}

At a concentration of $10 \mathrm{mg} / \mathrm{mL}$ and a temperature of $25^{\circ} \mathrm{C}$, the nanoparticle was suspended in deionized water for $90 \mathrm{~s}$. Then, the surface charge of anthocyanin loaded dextran nanoparticles was assessed through a zeta sizer Nano ZS (Malvern Panalytical, UK) (Tan et al., 2019).

\section{Composition of the functional groups via Fourier transform infrared (FTIR)}

Method of Tan et al. (2019) was applied. The samples used were anthocyanin extran, dextran and anthocyanin-loaded dextran nanoparticles. The samples were analysed using FTIR Spectrometer Spectrum RX I (Perkin Elmer, USA). All spectra were detected in the wavenumber range of $4000-400 \mathrm{~cm}^{-1}$.

\section{Anthocyanin release property}

Anthocyanin-loaded dextran nanoparticles were suspended in phosphate buffer solution at a concentration of $10 \mathrm{mg} / \mathrm{mL}, \mathrm{pH}$ 7.4. The solution was agitated on an orbital shaker at a rotational speed of $120 \mathrm{rpm}, 37^{\circ} \mathrm{C}$. At predetermined time gaps of 1, 2, 4, 8, 12, 24, 36, 48, and $60 \mathrm{~h}, 1 \mathrm{~mL}$ of sample was taken and analyzed through UV/Vis spectrophotometer. The amount of anthocyanin released was determined at $517 \mathrm{~nm}$ and quantified according to a calibration curve. The calibration curve was prepared by using $C$. ternatea derived anthocyanin as reference molecule. The standards were prepared at a range of concentrations $(0-$ $1000 \mu \mathrm{g} / \mathrm{mL}$ ) by performing two-fold dilution. The analysis was performed in three replicate on separate occasions (Shaikh et al., 2009).

\section{Drug encapsulation efficiency.}

The anthocyanin-loaded dextran nanoparticles were dissolved in PBS. Then, the solution was submerged for $15 \mathrm{~min}$ in the ultrasonic ice bath. The resulting solution was centrifuged at 20,000 $\mathrm{rpm}$ for $15 \mathrm{~min}$. After that, the supernatant was quantified spectrophotometrically at $517 \mathrm{~nm}$ via $\mathrm{UV} / \mathrm{V}$ is spectrophotometer. The encapsulation 
efficiency was measured according to the following equation;

$$
\mathrm{EE}(\%)=\frac{\text { Total amount of anthocyanin }- \text { free anthocyanin }}{\text { Total amount of anthocyanin taken for loading study }}
$$

\section{Stability of nanoparticles}

The stability of anthocyanin-loaded dextran nanoparticle and free anthocyanin was studied at different temperature $\left(37^{\circ} \mathrm{C}, 50^{\circ} \mathrm{C}, 80^{\circ} \mathrm{C}\right)$ and light condition. The sample was placed at $37^{\circ} \mathrm{C}, 50^{\circ} \mathrm{C}$ and $80^{\circ} \mathrm{C}$ for $24 \mathrm{~h}$. The absorbance of all samples, before and after exposure to different conditions, were analysed at a wavelength of $362 \mathrm{~nm}$.

\section{Anti-proliferative activity of anthocyanin- loaded dextran nanoparticles \\ Cell culture}

Liver cancer cells (HepG2) were maintained in minimal essential medium (MEM) $[10 \%(\mathrm{v} / \mathrm{v})$ fetal bovine serum and $1 \%(\mathrm{v} / \mathrm{v})$ penicillin $(100$ $\mathrm{U} / \mathrm{mL})$ ]. In a closed incubation chamber, the cell culture was exposed to $5 \% \mathrm{CO}_{2}$ atmosphere at $37^{\circ} \mathrm{C}$ for $48 \mathrm{~h}$. Next, the attached cells were trypsinated and centrifuged at 3,900 $\mathrm{rpm}$ for 10 min. The cells were counted using a hemocytometer and diluted to get a concentration of $7 \times 10^{3}$ cells $/ \mathrm{mL}$. Next, $100 \mu \mathrm{L}$ of HepG 2 cells were seeded in 96 well plates and further incubated at a similar temperature and $\mathrm{CO}_{2}$ atmosphere as mentioned above. This procedure was performed to attach the cells to the bottom of the wells.

\section{Analysis of cell proliferation}

MTT assay was demonstrated to evaluate the viability of the cells treated with anthocyaninloaded dextran nanoparticle, free anthocyanin and blank nanoparticle (dextran nanoparticles without loading of anthocyanin). At first, $100 \mu \mathrm{L}$ of the yellow color MTT solution $(1 \mathrm{~mL}$ in $9 \mathrm{~mL}$ fresh medium) was added to each well and further incubated at $37^{\circ} \mathrm{C}$ for $24 \mathrm{~h}$. After that, $100 \mu \mathrm{L}$ of DMSO was pipetted to each well to dissolve the formazan crystal. The presence of viable cells was determined by the formation of a purple color of formazan crystals. Subsequently, the absorbance was quantified at $570 \mathrm{~nm}$ via a microplate reader (Varioskan Lux, Thermo Scientific, Finland). The mixture with blank nanoparticle was used to set blank. The cell viability was verified by using the following equation;

Cell viabilitty $(\%)=\frac{(\text { Absorbance of sample }- \text { Absorbance of blank })}{(\text { Absorbance of control - Absorbance of blank })} \times 100 \%$

The cell death was calculated by subtracting the cell viability (\%) from 100\%. A graph of cell death $(\%)$ versus test substance concentration was plotted.

\section{RESULTS AND DISCUSSION}

\section{Characterization of anthocyanin nanoparticles}

Dextran was applied to entrap anthocyanin in the form of nanoparticles. Dextran with tremendous aqueous solubility, biocompatibility and nonfouling properties is broadly employed as an encapsulant polymer for drug nanoparticles. Figure 1 shows the morphology of the anthocyanin-loaded dextran nanoparticles under TEM view. The nanoparticles appeared in spherical, with a size range from 40 to $110 \mathrm{~nm}$. By average, the particle size was $45.5 \pm 11 \mathrm{~nm}$. As reported by Mudshinge et al. (2011), the nanoparticle solution has particles ranging from $1-200 \mathrm{~nm}$ in diameter, although the nanoparticles may not distribute evenly in the solution. In addition, there was no agglomeration observed in Figure 1. One of the factors contributed to such observation was the addition of pluronic F-127 as a stabilizer. This chemical was added to prevent the agglomeration of the nanoparticles by reducing their surface energy (Lee at al., 2015).

Surface charge of the nanoparticles draws a thin layer of ions of the different charge to the nanoparticle surface. The combined ion layers travel together with the nanoparticles as it disperses throughout the solution. In this experiment, both anthocyanin-loaded nanoparticles and dextran have negative charges i.e. $-4.39 \mathrm{mV}$ and $-4.46 \mathrm{mV}$, respectively. The high absolute value of zeta potential shows that high electric charge on the surface of the nanoparticles incorporated with a drug. This event causes strong repellent forces among particles, hence avoids the agglomeration event of the nanoparticles in buffer solution. In contrast, nanoparticles with low zeta potential will be 
stacked accordingly to van der Walls inter-particle pulls. This event might be due to the weak ionic strength of the nanoparticles. Leary (2012) stated that small changes in ionic strength and $\mathrm{pH}$ can lead to huge effects in zeta potential.

Figure 2 exhibits all spectra of anthocyaninloaded dextran nanoparticle, free anthocyanin and dextran under FTIR observation. First and foremost, the phenol $\mathrm{O}-\mathrm{H}$ stretch for dextran, free anthocyanin, and anthocyanin-loaded dextran nanoparticle are $3286.51 \mathrm{~cm}^{-1}, 3259.17 \mathrm{~cm}^{-1}$, and $3310.74 \mathrm{~cm}^{-1}$, respectively. The $\mathrm{C}-\mathrm{H}$ stretch absorptions can be seen just below $3000 \mathrm{~cm}^{-1}$ for all samples. These wavelengths symbolize the presence of saturated carbons. The peak above $3000 \mathrm{~cm}^{-1}$ is in fact from a hydroxyl group because of the broad gap that produced above the wavelength. Data of the functional groups for all test samples were summarized in Table 1. Merlic et al. (2001) reported that this functional group represents the presence of the exchangeable of protons. It was noticed that between the 3 samples, the strongest hydroxyl group absorption was noticed in free anthocyanin at $3259.17 \mathrm{~cm}^{-1}$ compared to dextran $\left(3286.51 \mathrm{~cm}^{-1}\right)$ and anthocyanin-loaded dextran nanoparticles $\left(3310.74 \mathrm{~cm}^{-1}\right)$. It was reported by Gundala and Aneja (2000) that O-H groups serve as electron acceptors or hydrogen atom donors and reduce the leftover reactive oxygen species. Owing to the metal chelating and stabilizing properties of some phenolic compounds, free radicals are removed, thereby conferring strong antioxidant activity.

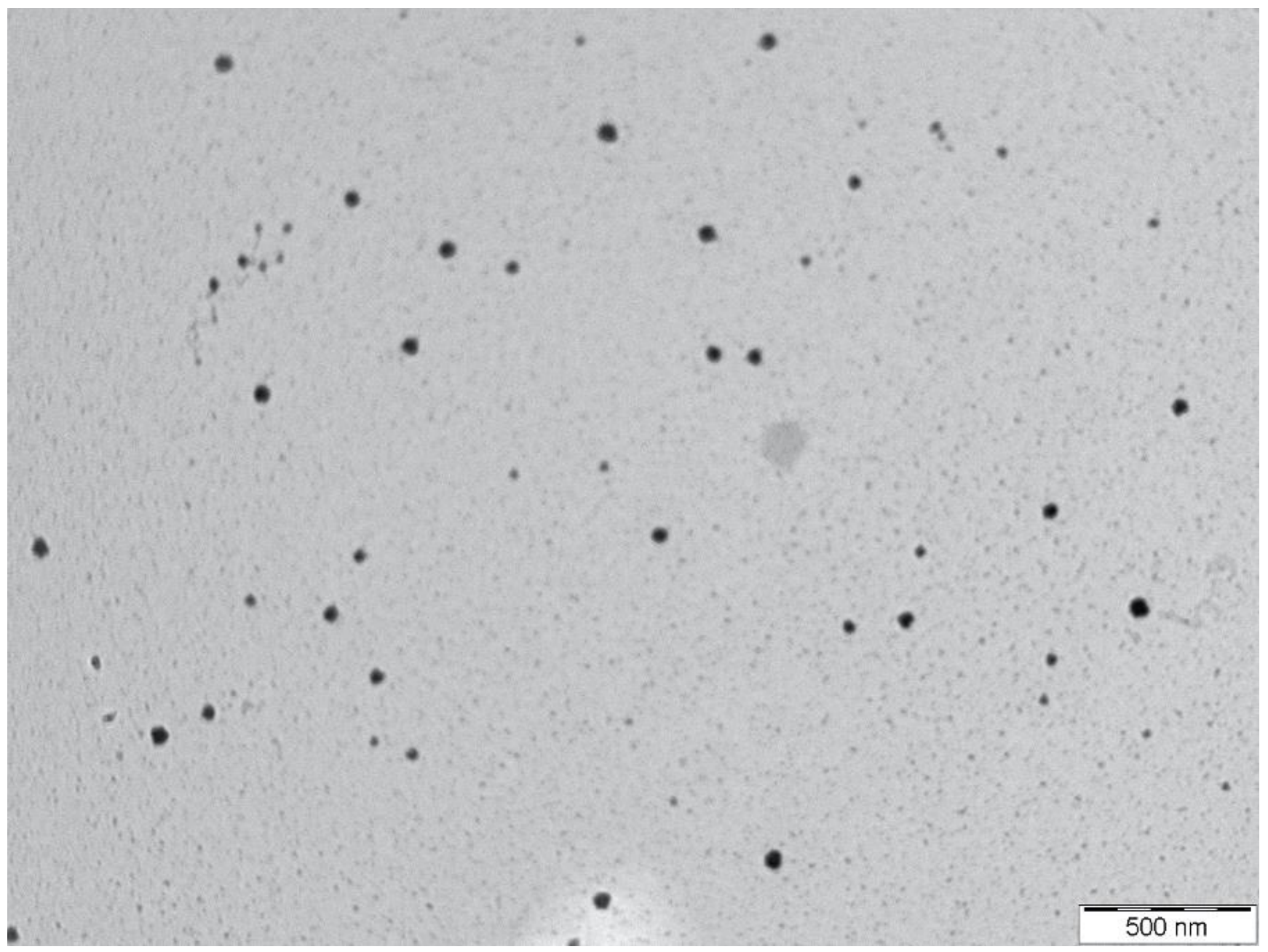

Figure 1. TEM micrograph of anthocyanin-loaded dextran nanoparticle 


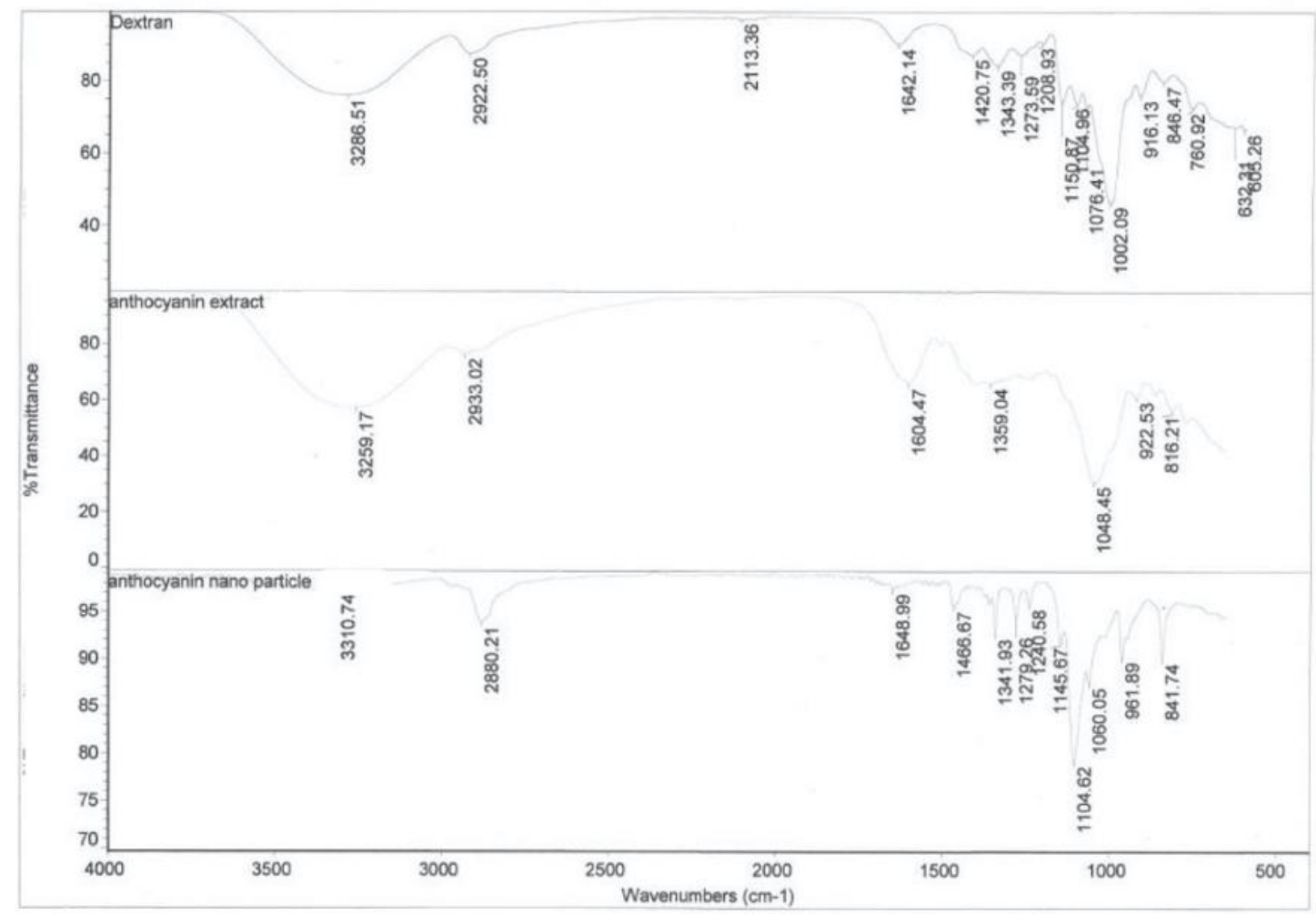

Figure 2. Functional groups in (A) dextran, (B) free anthocyanin, and (C) anthocyanin-loaded dextran nanoparticle based on FTIR analysis.

\section{Release property of anthocyanin nanoparticles}

The release of anthocyanin from the dextran nanoparticles was observed at $37^{\circ} \mathrm{C}$ for $60 \mathrm{~h}$ (Figure 3). The amount of anthocyanin released in the medium was quantified based on the standard calibration with a $R_{2}$ value of 0.928 . As observed, the release was slow and incremental throughout the experimental period. The anthocyanin release profile showed zero burst impact. The release of drug achieved plateau phase at $48 \mathrm{~h}$ with a total release of $48.35 \%$. The drug released has reached the minimum effective concentration as the time increased. The results showed that most of the anthocyanin was found in the internal structure of the polymer matrix. The drug release behaviour of nanoparticles is related to the polymer matrix structure and also the physicochemical properties of the drug (Soppimath et al., 2001). The slow drug release property of nanoparticles decreases the degradation of the drug, thus prolonging its bioavailability (Aditya et al., 2015). In overall, the drug release property of anthocyanin-loaded dextran nanoparticles provide a constant drug release to the medium, without a rapid increase of drug concentration. The results also showed that dextran is an ideal polymer to encapsulate anthocyanins, which retard the burst release effect of anthocyanin to the test medium. This control release property is very important to ensure long term therapeutic effect.

\section{Drug encapsulation efficiency}

There was approximately $3.03 \%$ of encapsulation efficiency recorded by anthocyanin-loaded dextran nanoparticle. The percentage explained that $3.03 \%$ of anthocyanin was successfully entrapped in the polymeric dextran nanoparticle. Low concentration of anthocyanin inside the nanoparticle can reflect the low percentage of encapsulation efficiency. As reported by Lee $e t$ al. (2015), the increase in encapsulation efficiency is in line with the increase of drug concentration used in the formulation. The solubility of the polymer and use of co-solvent is also influenced the drug encapsulation efficiency (Bhalekar et al., 
2009). Besides, the low encapsulation efficiency can be caused by low stirring speed during the nanoparticles synthesis. The encapsulation efficiency can be improved by increasing the stirring speed, in order to create high shear stress which facilitates the dispersion of viscous droplets of anthocyanins (Lee et al., 2015).

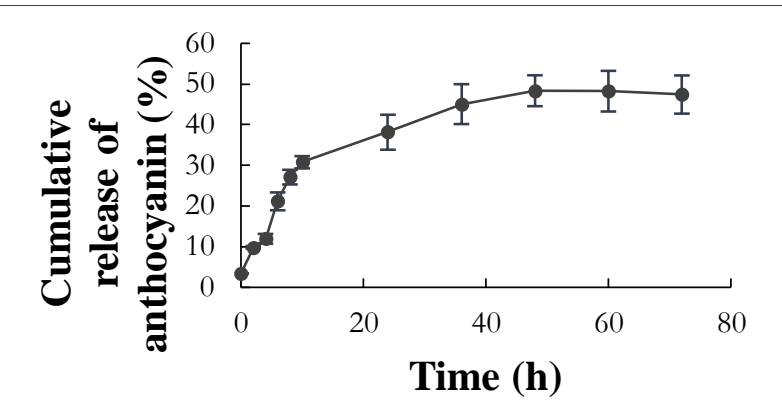

Figure 3. Drug release study of anthocyanin loaded nanoparticles

\section{Stability of anthocyanin-loaded dextran nanoparticle}

A comparison of the pigment stability between anthocyanin-loaded nanoparticles and free anthocyanin was exhibited in Table 1. In general, both samples were in blue and more stable in dark condition (amber glass). However, with light exposure, the color of the free anthocyanin started to fade and recorded lower pigment stability index than the anthocyanin-loaded nanoparticles. The similar events were observed at $37^{\circ} \mathrm{C}$ and $50^{\circ} \mathrm{C} \quad(P<0.05)$. Hence, the anthocyanin-loaded nanoparticles were significantly stable than free anthocyanin. At $80^{\circ} \mathrm{C}$, the stability indexes for both samples were relative $(P>0.05)$.

The pigment stability of anthocyanin is reliant on several factors including $\mathrm{pH}$, light, temperature, as well as its structure (Laleh et al., 2006). In this experiment, two factors were considered i.e. light exposure and also temperature. For instance, the exposure of anthocyanin extracted from $C$. ternatea to light can destruct the anthocyanin pigments. Our results were in line with Bakhshayeshi et al. (2006). Under light exposure, the flavylium cation is increasingly constructed, however, in dark condition, the amount of chalcone denotes higher than flavylium cation. Generally, flavylium cation is a basic structure of anthocyanins which engages with the red color. Thus, the active construction of flavylium cation drives into color changes in $C$. ternatea, which originally in purple hue color. Our results showed there was better color or pigment stability index obtained from anthocyanin-loaded dextran nanoparticle. In conclusion, the dextran that encapsulated the anthocyanin significantly improved the stability of anthocyanin itself.

The co-pigment complexes are exothermic and sensitive to temperature (Roobha et al., 2011). It has been shown that the rise in temperature shifted the anthocyanin equilibrium towards the chalcone form. Hence as the temperature increase, there will be a large decrease in absorbance. The anthocyanin-loaded dextran nanoparticle was more stable due to the selfagglomeration of anthocyanins that prevents the hydration of anthocyanins, preserves the flavylium cation and enhances the stability of anthocyanins (Gris et al., 2009).

\section{Analysis of cell proliferation}

The anti-proliferative efficiency of anthocyaninloaded dextran nanoparticles was tested against the human liver carcinoma cells, HepG2 (Figure 4). The free anthocyanin and encapsulated anthocyanin were added to the culture medium of HepG2 cells and incubated for $24 \mathrm{~h}$. The MTT assay was done to evaluate the anti-proliferative potential of the anthocyanin-loaded nanoparticles and free anthocyanin. The HepG2 cells treated with the anthocyanin-loaded dextran nanoparticles showed significantly higher cell death compared to free anthocyanin at a concentration range from 15.6-125.0 $\mu \mathrm{g} / \mathrm{mL}$. For free anthocyanin, the highest cell death recorded was $33.4 \%$ at $250.0 \mu \mathrm{g} / \mathrm{mL}$. In contrast, by nanoencapsulation with dextran, the highest cell death recorded was $67.3 \%$ at $62.5 \mu \mathrm{g} / \mathrm{mL}$. Nanoencapsulation with dextran significantly improved the anti-proliferative significantly improved the anti-proliferative activity of $C$. ternatea derived anthocyanins. It is noticeable that the cell death recorded was reduced when the anthocyaninloaded dextran nanoparticles were tested at 125.0 and $250.0 \mu \mathrm{g} / \mathrm{mL}$. This is due to the agglomeration of nanoparticles when the dissolved nanoparticles were tested at high concentration. Agglomeration refers to adhesion of the nanoparticles to each other by weak forces leading to the formation of sub-microparticles (Pellegrino et al., 2017). The agglomerated 
particles reduce the absorption of the drug to the cancer cells, thus reducing the therapeutic efficiency (Limbach et al., 2005; Lankoff et al., 2012). The agglomeration of the nanoparticles can be avoided by addition of surfactant type stabilizing agents (Mehta et al., 2009).

The formation of nanoparticles helps to sustain the release of the anthocyanin and thus improving the drug absorption to the cancer cells.. Nano-encapsulation with dextran prolongs the bioavailability of anthocyanins. The nanoparticles also lower the degradation of anthocyanin in cancer cells treatment. Dextran might increase the bioavailability of anthocyanins, thus enhances its anti-cancer properties. Similar observation was reported by other researchers. A study reported by Esmatabadi et al. (2015) signified a comparison between raw curcumin and curcumin grafteddendrosome nanoparticles on cell viability of SW480 colon carcinoma and Huh7 hepatome cells after incubation time for $48 \mathrm{~h}$. The treatment of cell lines with curcumin loaded-dendrosome significantly reduced cell viability of SW480 and Huh7 cells more than free curcumin. Cell sensitivity to curcumin was greatly increased once the cells were treated with curcumin loadeddendrosome. As a result, curcumin loadeddendrosome increased in bioavailability than free curcumin. Besides, the loading of cisplatin in dextran nanoparticles also improved the antitumor activity og metastatic breast cancer.

Table 1. Anthocyanin stability index at different temperature and light conditions.

\begin{tabular}{lccccc}
\hline & \multicolumn{5}{c}{ Colour stability index } \\
\cline { 2 - 6 } & Amber glass & Light glass & $\mathbf{3 7 ^ { \circ } \mathbf { C }}$ & $\mathbf{5 0}{ }^{\circ} \mathbf{C}$ & $\mathbf{8 0}^{\circ} \mathbf{C}$ \\
\hline $\begin{array}{l}\text { Anthocyanin- } \\
\text { loaded dextran } \\
\text { nanoparticle }\end{array}$ & 0.7675 & 0.7540 & 0.9151 & 0.58688 & 0.3522 \\
\hline
\end{tabular}

$\begin{array}{llllll}\text { Free anthocyanin } & 0.6819 & 0.4580 & 0.5952 & 0.40606 & 0.3459\end{array}$

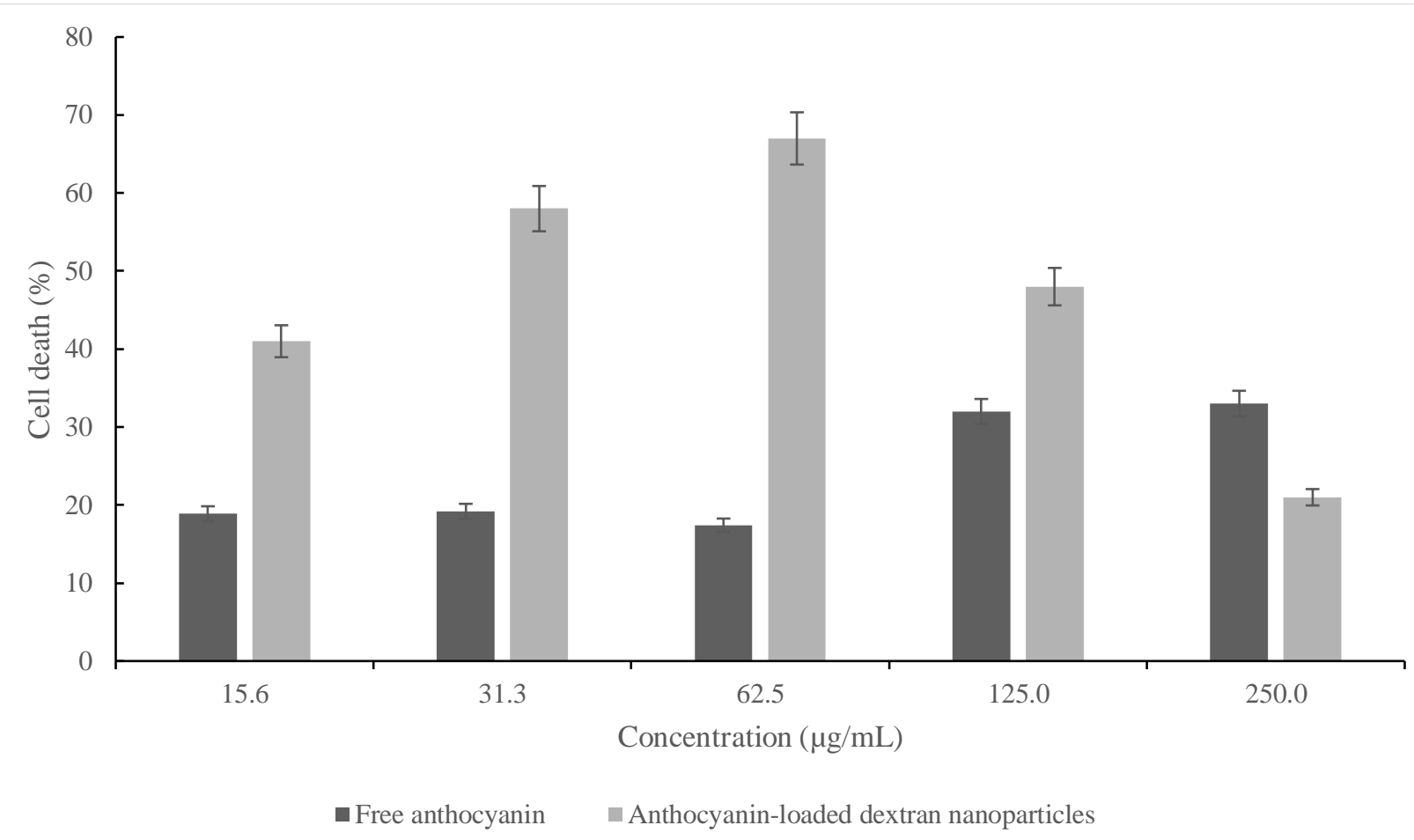

Figure 4. Cell death analysis of human liver carcinoma cells, HepG2 after incubated with free anthocyanin and anthocyanin-loaded dextran nanoparticles. 


\section{CONCLUSION}

A competent nanoparticle-based drug delivery system which involved anthocyanin and dextran was successfully developed. The nanoencapsulation of $C$. ternatea derived anthocyanins with dextran also significantly improved the light and heat stabilities of the anthocyanins. The antiproliferative activity of the anthocyanin-loaded dextran nanoparticles were significantly better than free anthocyanins on HepG2 cell lines. Further investigation must be conducted to test the anti-proliferative activity of the anthocyaninloaded dextran nanoparticles on more cancer cell lines to explore their pharmaceutical potential.

\section{ACKNOWLEDGEMENTS}

This study is funded by Ministry of Higher Education under the Fundamental Research Grant Scheme (FRGS/1/ 2017/STG03/ UNIKL/02/1).

\section{REFERENCES}

Aditya, N. P., Aditya, S., Yang, H., Kim, H. W., Park, S. O. \& Ko, S. 2015. Co-delivery of hydrophobic curcumin and hydrophilic catechin by a water-in-oil-in-water double emulsion. Food Chemistry 173: 7-13.

Bakhshayeshi, M. A., Khayami, M., Heidari, R. \& Jamei, R. 2006. The effects of light, storage temperature, $\mathrm{pH}$ and variety on stability of anthocyanin pigments in four Malus varieties. Pakistan Journal of Biological Sciences 9: 428-433.

Bhalekar, M. R., Pokharkar, V., Madgulkar, A., Patil, N., \& Patil, N. 2009. Preparation and evaluation of miconazole nitrateloaded solid lipid nanoparticles for topical delivery. American Association of Pharmaceutical Scientists PharmSciTech 10(1): 289-296.

Bhatia, M., Chahal, J. \& Gupta, S. 2014. Analgesic and antiInflammatory activities of Clitoria ternatea linn. leaves extract on rat model. International Journal Pharmacentical Sciences Research 5(2): 600-606.

Braga, C. B., Kido, L. A., Lima, E. N., Lamas, C. A., Cagnon, V. H., Ornelas, C. \& Pilli, R. A. 2020. Enhancing the anticancer activity and selectivity of Goniothalamin using $\mathrm{pH}$-sensitive acetalated dextran (Ac-Dex) nanoparticles: A promising platform for delivery of natural compounds. ACS Biomaterials Science and Engineering 6(5): 2929-2942.

Bray, F., Ferlay, J., Soerjomataram, I., Siegel, R. L., Torre, L.A. \& Jemal, A. 2018. Global cancer statistics 2018: GLOBOCAN estimates of incidence and mortality worldwide for 36 cancers in 185 countries. CA: A Cancer Journal for Clinicians 68(6): 394-424.
Esmatabadi, M. J., Sarkandi, M. K., Zadeh, H. M., Khaledi, G., Montazeri, M., Shekarabi, H. S. Z., Hormoz, Y. A. \& Asgari, E. A. 2015. Comparative evaluation of curcumin and curcumin loaded-dendrosome nanoparticle effects on the viability of SW480 colon carcinoma and Huh7 hepatoma cell. Research Journal of Pharmacognosy 2(3): 9-16.

Gérard, V., Ay, E., Morlet-Savary, F., Graff, B., Galopin, C., Ogren, T. \& Lalevée, J. 2019. Thermal and photochemical stability of anthocyanins from black carrot, grape juice, and purple sweet potato in model beverages in the presence of ascorbic acid. Journal of Agricultural and Food Chemistry 67(19), $5647-5660$

Gris, E. F.,Fereira, E. A.,Falcao, L. D. \& Bordignon-Luiz, M. T. T. 2009. Caffeic acid copigmentation of anthocyanins from cabernet sauvignon grape extracts in model system. Food Chemistry 100: 1289-1296.

Gundala, S. R. \& Aneja, R. 2000. Piper betel leaf: a reservoir of potential xenohormetic nutraceuticals with cancer-fighting properties. American Association for Cancer Research 7(5): 47786.

Hwang, Y. P., Choi, J. H., Choi, J. M., Chung, Y. C. \& Jeong, H. G. 2011. Protective mechanisms of anthocyanins from purple sweet potato against tert-butyl hydroperoxideinduced hepatotoxicity. Food and Chemical Toxicology 49(9): 2081-2089.

Karaoglan, H. A., Keklik, N. M. \& Isıkl, N. D. 2019. Degradation kinetics of anthocyanin and physicochemical changes in fermented turnip juice exposed to pulsed UV light. Journal of Food Science and Technology 56(1): 30-39.

Khan, I., Saeed, K. \& Khan, I. 2019. Nanoparticles: Properties, applications and toxicities. Arabian Journal of Chemistry 12(7), 908-931.

Kong, J. M., Chia, L. S., Goh, N. K., Chia, T. F. \& Brouillard, R. 2003. Analysis and biological activities of anthocyanins. Phytochemistry 64: 923-933.

Laleh, G. H, Frydoonfar H., Heidary R., Jameei R. \& Zare S. 2006. The effect of light, temperature, $\mathrm{pH}$ and species on stability of anthocyanin pigments in four Berberis species. Pakistan Journal of Nutrition 5(1): 90-21.

Leary, J. F. 2012. The importance of zeta potential for drug/gene delivery in nanomedicine. https://nanohub.org/resources/ 13630.

Lee, W. H., Bebawy, M., Loo, C. Y., Luk, F., Mason, R. S. \& Rohanizadeh, R. 2015. Fabrication of curcumin micellar nanoparticles with enhanced anti-cancer activity. Journal Biomedical Nanotechnology 10:1-13.

Leong, C. R., Kamarul Azizi, M. A., Taher, M. A., Wahidin, S., Lee, K. C., Tan, W. N. \& Tong, W. Y. 2017. Anthocyanins from Clitoria ternatea attenuate food-borne Penicillium expansum and its potential application as food biopreservative. Natural Product Sciences 23(2): 125-131.

Merlic, C. A., Fam, B. C., \& Miller, M. M. 2001. WebSpectra: Online NMR and IR for students. Journal of Chemical Education 78(1): 118.

Mohanraj, V. J. \& Chen, Y. 2006. Nanoparticles - A review. Tropical Journal of Pharmaceutical Research 5(1): 561-573.

Mudshinge, S. R., Deore, A. B., Patil, S. \& Bhalgat, C. M. 2011. Nanoparticles: emerging carriers for drug delivery. Saudi Pharmacentical Journal 19(3): 129-141.

Oliver, S., Yee, E., Kavallaris, M., Vittorio, O. \& Boyer, C. 2018. Water soluble antioxidant dextran-quercetin conjugate with potential anticancer properties. Macromolecular Bioscience 18(4): 1700239.

Parry, J., Su, L., Moore, J., Cheng, Z., Luther, M., Rao, J. N., Wang, J. Y. \& Yu, L. L. 2006. Chemical compositions, antioxidant capacities, and antiproliferative activities of selected fruit 
seed flours. Journal of Agricultural and Food Chemistry 54(11): 3773-3778.

Prior, R. L. \& Wu, X. 2006. Anthocyanins: structural characteristics that result in unique metabolic patterns and biological activities. Free Radical Research 40(10): 1014-1028.

Ray, H. J., Lundy, S., Eriksen, C. \& Kalicki, B. 2009. Anthocyanins. Pennington Nutrition Series 1: 1-4.

Renis, M., Calandra, L., Scifo, C., Tomasello, B., Cardile, V., Vanella, L., Bei, R., La Fauci, L. \& Galvano, F. 2008. Response of cell cycle/stress-related protein expression and DNA damage upon treatment of $\mathrm{CaCo} 2$ cells with anthocyanins. British Journal of Nutrition 100(1): 27-35.

Roobha, J. J., Saravanakumar, M., Aravindhan, K. M. \& Devi, P. S. 2011. Effect of light, temperature, $\mathrm{pH}$, on stability of anthocyanin pigments in Musa acuminate bract. Research in Plant Biology 1(5): 5-12.

Shih, P. H., Yeh, C. T. \& Yen, G. C. 2007. Anthocyanins induce the activation of phase II enzymes through the antioxidant response element pathway against oxidative stress-induced apoptosis. Journal of Agricultural and Food Chemistry 55(23): 9427-9435.

Shaikh J., Ankola, D. D., Beniwal, V., Singh, D., \& Kumar, M. R. 2009. Nanoparticle encapsulation improves oral bioavailability of curcumin by at least 9-fold when compared to curcumin administered with piperine as absorption enhancer. European Journal of Pharmaceutical Sciences 37(3): 223-230.

Soppimath, K. S., Aminabhavi, T. M., Kulkarni, A. R. \& Rudzinski, W. E. 2001. Biodegradable polymeric nanoparticles as drug delivery devices. Journal of Controlled Release 70(1-2): 1-20.

Tan, W. N., Leong, C. R., Venoth, A., Judy Loo, C. Y., Lee, W. H., Fahmi Asyadi, M. Y., Mohd Azizan, M. N. \& Tong, W. Y. 2019. Sustained release geraniol nanoparticles inhibit human axillary odor-causing bacteria. Arabian Journal for Science and Engineering 44: 103-109.

Telegeev, G., Kutsevol, N., Chumachenko, V., Naumenko, A., Telegeeva, P., Filipchenko, S. \& Harahuts, Y. 2017. Dextran-Polyacrylamide as matrices for creation of anticancer nanocomposite. International Journal of Polymer Science 48: 3-9.

Thanh, V. T., Tran, N. Y., Linh, N. T. V., Vy, T. A. \& Thanh, T. 2020. Application of anthocyanin natural colors from Butterfly Pea (Clitoria ternatea L.) extracts to cupcake. MS\&E 736(6): 062014.

Tong, W. Y., Leong, C. R., Tan, W. N., Khairuddean, M., Zakaria, L. \& Ibrahim, D. 2017. Endophytic Diaporthe sp. ED2 produce a novel anti-candidal ketone derivative. Journal of Microbiology and Biotechnology 27(6):1065-1070.

Vittorio, O., Brandl, M., Cirillo, G., Kimpton, K., Hinde, E., Duong, H. T. \& Parmar, A. 2016. Dextran-Catechin conjugate: An anticancer nano-modified natural compound targeting copper metabolism in neuroblastoma. Oncotarget 7(30): 47479-47493.

Wang, L. S. \& Stoner, D. G. 2008. Anthocyanins and their role in cancer prevention. Cancer Letters 269: 281-290.

Wang, S. Y. \& Jiao, H. 2000. Scavenging capacity of berry crops on superoxide radicals, hydrogen peroxide, hydroxyl radicals, and singlet oxygen. Journal of Agricultural and Food Chemistry 48(11): 5677-5684.

Windchester, D. P., Stewart, A. K., Phillips, J. L. \& Ward, E. E. 2010. The national cancer data base: past, present, and future. Annals of Surgical Oncology 17(1): 4-7.

Yang, W., Kortesniemi, M., Ma, X., Zheng, J. \& Yang, B. 2019. Enzymatic acylation of blackcurrant (Ribes nigrum) anthocyanins and evaluation of lipophilic properties and antioxidant capacity of derivatives. Food Chemistry 281: 189196.
Zununi, V. S., Fathi, N., Samiei, M., Maleki Dizaj, S. \& Sharifi, S. 2019. Targeted cancer drug delivery with aptamerfunctionalized polymeric nanoparticles. Journal of Drug Targeting 27(3): 292-299. 